\title{
Frauenförderung an der TFH Wildau - Projektarbeit in der Lehre -
}

\author{
Prof. Dr. rer. pol. Helga Meyer
}

\section{Zusammenfassung}

FIPRO - Frauen im Projektmanagement ist ein Forschungs- und Lehrprojekt der Technischen Fachhochschule Wildau. Frauen in Projektfunktionen sind Gegenstand der Forschungsarbeit. Das Projekt selbst ist eine aktive Maßnahme zur Förderung von Frauen während ihres Studiums, um die Position der Studentinnen im Hinblick auf ihren späteren Berufseinstieg und auch bezogen auf spätere Karrieremöglichkeiten im Sinne der Chancengleichheit zu stärken.

\section{Frauenförderung im Studium - Weshalb?}

Frauenförderung heißt, durch bestimmte Aktionen einen Beitrag zur beruflichen Chancengleichheit von Frauen und Männern zu leisten. Betriebliche Frauenförderung soll neben Erschließung und Nutzung weiblichen Humankapitals durch freiwillige positive Aktionen auch durch Veränderung der Arbeitsorganisation einen Beitrag zur beruflichen Chancengleichheit von Frauen und Männern bewirken [1]. Es geht nicht darum, weibliche Arbeitskräfte ungerechtfertigt zu bevorzugen, sondern ihnen mehr und qualifiziertere Möglichkeiten im Erwerbsleben zu bieten. Im Idealfall handelt es sich nicht nur um einseitige Maßnahmen für Frauen, sondern gleichzeitig auch um Aktionen für Männer, um deren Einstellungen zu verändern.

An den Hochschulen studieren immer mehr Frauen, und zwar vor allem in betriebswirtschaftlichen, aber auch in ingenieurwissenschaftlichen Studiengängen. Schon seit mehreren Jahren sind ca. 40 Prozent aller Studenten Frauen. Vor allem der Anteil der Absolventinnen in den Wirtschaftswissenschaften hat sich auf hohem Niveau bei über 30 Prozent stabilisiert [2]. Es ist die Aufgabe der Hochschulen, dafür zu sorgen, daß diese zukünftigen Absolventinnen auf dem Arbeitsmarkt die gleichen Chancen erhalten wie ihre männlichen Kollegen. Diese Gleichberechtigung bezieht sich sowohl auf den Berufseinstieg als auch auf spätere Karrieremöglichkeiten. Denn Frauen mit Abitur/Fachhochschul-/Hochschulausbildung sind mindestens ebenso qualifiziert und leistungsmotiviert wie Männer. Aus volks- und betriebswirtschaftlicher Sicht ist es daher ökonomisch, dieses Potential zu berücksichtigen [3].

Chancengleichheit stellt sich nicht von selbst ein, wie empirische Untersuchungen immer wieder zeigen. Die Daten der folgenden Tabelle verdeutlichen, daß Frauen in Managementpositionen in der Bundesrepublik immer noch unterrepräsentiert sind.
Die Daten der Bundesrepublik Deutschland weisen außerdem daraufhin, daß gerade in der Industrie, einem Wirtschaftszweig, in dem traditionell häufig Projekte durchgeführt werden, Frauen selten vertreten sind. Hinzu kommt, daß im Zeichen wachsender Arbeitslosigkeit ohnehin Rückschritte zu befürchten sind. Erste Untersuchungen aus England und Australien belegen diese Befürchtungen bereits. Eine empirische Studie des Institute of Management and Remuneration Economics ergab. daß der Anteil der Frauen in Managementpositionen insgesamt bis 1993 stetig zunahm bis auf 10,2\%. Dagegen fiel der Anteil 1994 auf 9,5\% zurück [4].

\begin{tabular}{|l|c|c|c|}
\hline Branche & $\begin{array}{c}\text { Unteres } \\
\text { Management }\end{array}$ & $\begin{array}{c}\text { Mittleres } \\
\text { Management }\end{array}$ & $\begin{array}{c}\text { Top- } \\
\text { Management }\end{array}$ \\
\hline Industrie & 5,5 & 2,7 & 2,8 \\
\hline $\begin{array}{l}\text { Banken und } \\
\text { Versicherungen }\end{array}$ & 8,7 & 3,6 & 0,8 \\
\hline Handel & 10,5 & 5,3 & 5,1 \\
\hline
\end{tabular}

Abb. 1: Anteil von Frauen am Management im Verhältnis zum Männeranteil in der Bundesrepublik Deutschland (in Prozent) |5|

Auf die Darstellung weiteren Zahlenmaterials sei an dieser Stelle verzichtet. Es besteht weitestgehend Einigkeit darüber, daß in der Bundesrepublik eine berufliche Chancengleichheit von Frauen und Männern vor allem im Bereich der Führungskräfte bislang nicht ausreichend realisiert ist.

\section{Erklärungsansätze}

Welche Erklärungs- und damit zugleich Handlungsansätze existieren? Verschiedene Ursachen werden auf drei Ebenen wirksam:

$\begin{array}{ll}\text { Eignungspotential von Frauen } & \text { - Qualifikation } \\ & \text { - Motivation } \\ & \text { - Persönlichkeit } \\ \text { Einstellungen gegenüber Frauen } & \text { - Vorurteile } \\ \text { Bestehende Strukturen } & \text { - Beruf und Familie } \\ & \text { - Arbeitsorganisation } \\ & \text { - Rollenvorbilder }\end{array}$

Eignungspotential von Frauen

Häufig werden Frauen und Männern bestimmte charakterologische Merkmale zugeschrieben, die sich voneinander unterscheiden und deshalb Frauen ein geringeres Eignungspotential für Führungspositionen attestieren. Dabei geht es weniger um formale Qualifikationen, die in Form von Zeugnisnoten nachweisbar sind. Vielmehr spielen sogenannte Persönlichkeitsmerkmale eine Rolle. Die Personalwirtschaft kennt auch den Begriff überfachliche Qualifikation, den die Autorin an dieser Stelle 
bevorzugen möchte. Diese beiden Begriffspaare weisen bereits auf die Diskussion hin, inwieweit Führungsfähigkeit erlernbar ist. Die Literatur kennt dazu ganz unterschiedliche Auffassungen. Es existieren Studien, die Kovarianzen zwischen bestimmten Persönlichkeitsmerkmalen und Managementeffizienz belegen [6]. Persönlichkeitseigenschaften, die häufig bei erfolgreichen Führungskräften identifiziert werden, sind Klugheit, Initiative, Selbstsicherheit, Entscheidungsfreude, Durchsetzungsfähigkeit. Dieser Erklärungsansatz zielt darauf ab, daß Führungsfähigkeit eben nicht vorwiegend erlernbar ist.

Auf vergleichbarer Ebene argumentieren Vertreter/innen von Fraueninteressen, die ebenfalls den Frauen bestimmte Eigenschaften zuschreiben, wie z. B. Teamfähigkeit, die wiederum Frauen besonders für Führungsaufgaben qualifizieren. Diese Argumentation beruht auf dem Konzept des „weiblichen Arbeitsvermögen“, welches auch auf die einfache Formel „Das Tun bestimmt das Sein“ reduziert werden kann [7]. Sie birgt jedoch einige Tükken, vor allem aber wird sie nach Meinung der Autorin dem eigentlichen Gedanken nach Chancengleichheit für Frauen und Männer nicht gerecht.

Eher von Bedeutung sind Forschungsergebnisse, die eine Professionalisierung auch im Bereich der Führungstätigkeiten nachweisen. Was spricht dagegen, das Treffen von Entscheidungen, Selbstsicherheit oder auch Teamfähigkeit zu trainieren? [8]

\section{Einstellungen gegenüber Frauen}

Zuvor wurden bereits Persönlichkeitsmerkmale benannt, die Frauen oder Männern zugeordnet werden. Diese Kategorisierung trifft jedoch keineswegs für alle Männer und alle Frauen zu. Weitere Vorurteile, die gegenüber Frauen aufgeführt werden, sind z. B. eine stärkere emotionale Steuerung der Frauen oder geringe Durchsetzungsstärke. Die Doppelbelastung durch Beruf und Familie ist ebenfalls ein Punkt, der nicht eben für den Einsatz von Frauen in Führungspositionen zu sprechen scheint. Existiert die Doppelbelastung noch nicht, so stellt sie zumindest bis zu einem bestimmten Alter immer noch eine potentielle Befürchtung dar. Denn irgendwann könnte die Frau ein Kind bekommen und dann erst einmal für längere Zeit ausfallen. Dieses Vorurteil wird sich wohl erst dann entschärfen, wenn Männer, die den Haushalt führen und Erziehungsurlaub nehmen, keine Ausnahme mehr sind. Auch wenn längst nachgewiesen ist, daß gerade Frauen, die in Führungspositionen arbeiten, sehr leistungsmotiviert sind und nach einer Geburt nur eine sehr kurze Familienpause einlegen [9].

\section{Bestehende Strukturen}

Hinsichtlich der Arbeitsorganisation werden zukünftig Arbeitszeitmodelle interessant, die es sowohl Männern als auch Frauen erlauben, Familie und Beruf sinnvoll zu vereinbaren, ohne daß Benachteiligungen in der beruflichen Entwicklung befürchtet werden müssen. Hierzu gibt es bereits in einigen Unternehmen Modelle. Der vermehrte Einsatz von Frauen in Führungspositionen schafft auch einen strukturellen Vorteil, denn das Problem der Persönlichkeit der Entscheider, die die Bewerber nach eigenen Mustern und Vorstellungen auswählen, ist im- mer noch ungelöst. Wenn Frauen vermehrt diese Entscheidungspositionen innehaben, können weibliche Nachwuchskräfte davon profitieren. Rollenvorbilder für motivierte weibliche Nachwuchskräfte können außerdem eine Stärkung z. B. des Selbstbewußtseins vermitteln.

Frauenförderung im Studium an der Technischen Fachhochschule Wildau integriert Aspekte aus allen drei Erklärungsansätzen. Wie dies in einem konkreten Projekt umgesetzt wird, zeigen die nachfolgenden Ausfuihrungen.

\section{Projekt FIPRO - Frauen im Projekt- management}

Seit dem Sommersemester 1994 läuft an der TFH Wildau im Fachbereich Betriebswirtschaft/Wirtschaftsinformatik das Projekt „FIPRO - Frauen im Projektmanagement“. Dieses Projekt wird unterstuitzt vom brandenburgischen Ministerium für Wissenschaft, Forschung und Kultur.

\section{Projektzielsetzung}

Das Projekt „FIPRO - Frauen im Projektmanagement" zeichnet sich durch eine Besonderheit aus: Es ist zugleich Forschungs- und Lehrprojekt und verfolgt daher Zielstellungen auf zwei Ebenen:

\section{- Ebene 1: Zielstellung des Forschungsprojekts}

Erkenntnisse gewinnen über Zugang, Qualifizierung und Arbeit in Projektfunktionen, insbesondere bezogen auf Frauen, z. B. über Hemmnisse und Barrieren, mögliche Personalentwicklungsmaßnahmen.

- Ebene 2: Zielstellung des Lehrprojektes

Das Forschungsprojekt ist eingebettet in die Lehre an der Technischen Fachhochschule Wildau mit dem Ziel, speziell Studentinnen zu fördern, indem ihnen bestimmte Qualifikationen vermittelt werden. Hierzu zählen Fertigkeiten im Bereich Projektmanagement, in empirischer Forschungsarbeit und Training in überfachlichen Fähigkeiten.

Im Rahmen des Projektes wird außerdem ein intensiver Kontakt zur Praxis gepflegt. Dadurch trägt das Projekt dazu bei, den Unternehmen positive Anreize für den vermehrten Einsatz von Frauen in Projektführungsfunktionen zu vermitteln.

\section{Projektmanagement}

Projekte erfolgreich bewältigen ist heutzutage einer der wesentlichen Erfolgsfaktoren für Unternehmen. Maßgeblich fuir den Erfolg von Projekten ist vor allem das Projektpersonal. Praxiskontakte, Tagungsbesuche und Seminare vermitteln den Eindruck, daß in Projektfuihrungsfunktionen bisher nur wenige Frauen vertreten sind. Unsere Gesellschaft kann es sich jedoch nicht leisten, die Hälfte der Menschheit in ihrem Potential unberüicksichtigt zu lassen [10]. Dies ist erst recht unter ökonomischen Gesichtspunkten wenig sinnvoll, wenn die Gesellschaft bereits sehr viel Geld in die Ausbildung dieser Personengruppe investiert hat.

Warum arbeiten bisher wenige Frauen in Projektfuihrungsfunktionen? Wie kann Frauen der Zugang zu diesen Funktionsbereichen ermöglicht werden? Diesen und 
anderen Fragen geht das Forschungs- und Lehrprojekt der Technischen Fachhochschule Wildau „FIPRO - Frauen im Projektmanagement" nach.

Projektfunktionen, insbesondere die Leitung eines Projektes, können eine wichtige Schlïsselrolle spielen, um Frauen den späteren Eintritt ins Management zu ermöglichen. Denn die Übernahme einer Projektleitung ist gewissermaßen eine Führungsfunktion auf Zeit. Projektmanagement ist daher eine wichtige Einstiegsposition für zukünftige Führungspositionen. Hinzu kommt, daß Projektmanagement in den letzten Jahren erheblich an Bedeutung gewonnen hat. Heute spielt Projektmanagement eine entscheidende Rolle bei den verschiedensten Aufgabenstellungen. So erledigen Großunternehmen bisher repetitiv bewältigte Aufgaben zunehmend projektorientiert, z. B. den Serienanlauf eines neuen Produktes.

Der Erfolg mittelständischer Unternehmen wird zunehmend durch die Fähigkeit bestimmt, Projekte zielorientiert und rationell zu planen und durchzuführen. Im Zeitalter der Kunder:orientierung erhöht sich nicht nur die Anzahl der Projekte, sondern auch die unternehmerische Bedeutung jedes einzelnen Projektes [11]. Mittelbetriebe müssen als Zulieferer Auftragsfertigung betreiben, eigene Produkte und Dienstleistungen müssen im Ausland vermarktet werden. Im Zuge des europäischen Binnenmarktes und der Öffnung in den Osten werden immer häufiger Joint venture-Vorhaben realisiert [12]. Sogar in der Verbandsarbeit kann Projektmanagement ein wichtiger Erfolgsfaktor sein. Die aktuelle Diskussion zeigt außerdem, Projektmanagement-Methoden bieten eine nützliche Unterstützung zur Bewältigung von Problemlösungsprozessen in der öffentlichen Verwaltung [13].

Zusammenfassend hat sich Projektmanagement fuir die Wirtschaft zu einer unverzichtbaren Führungskonzeption einschließlich der dazugehörenden Methoden entwickelt. Nur mit ihrer Hilfe können Organisationen ihre aktuellen und zukünftigen Aufgaben bewältigen, bei denen Werte wie Flexibilität, Selbstbestimmung in der Arbeit, Schnelligkeit, Termintreue, Kundennähe, geringe Kosten eine große Rolle spielen. Die Tendenz zu Kleinprojekten wird anhalten, innerhalb von Großprojekten und davon unabhängig. Verkürzte Lebenszyklen der Produkte erfordern häufige Entwicklungs- und Reorganisationsprojekte [14]. Aus der punktuellen Nutzung von Instrumenten auf der mittleren Ebene wird auch ein Führungskonzept der oberen Ebene: aus Projektmanagement entsteht Management by Projects [15].

Damit ist deutlich geworden: Projektmanagement-Qualifikationen sind heute unverzichtbar, wenn Absolventen betriebswirtschaftlicher und ingenieurwissenschaftlicher Studiengänge beruflich erfolgreich sein wollen. Eine Auswertung von Stellenausschreibungen für Projektpersonal von 1986 bis 1992 belegt den steigenden Bedarf der Wirtschaft an Projektmanagement-Qualifikationen [16]. Um so bedeutender wird die Notwendigkeit, daß auch Frauen in Projektschlïisselfunktionen arbeiten. Die Situation von Frauen im Projektmanagement, verglichen mit den Anforderungen der Wirtschaft im Hinblick auf Projektmanagement, läßt folgende These zu:
Gelingt es den Frauen nicht, in der Schlüsselqualifikation Projektmanagement und den entsprechenden Schlüsselfunktionen wie Projektleitung oder Projektcontrolling Fuß zu fassen, so werden sie eine wichtige Anschlußqualifikation versäumen. Hierdurch droht den Frauen über Jahre hinaus eine maßgebliche Beeinträchtigung ihrer Karriereentwicklung.

Wie kann Frauen der Zugang zu Schlïsselqualifikationen und Schlïsselfunktionen im Projektmanagement verschafft werden? Im einzelnen leiten sich daraus verschiedene Fragen ab:

- Wie wird Mann/Frau Projektleiter?

- Welche Laufbahnmodelle existieren für Projektleiter?

- Welche Faktoren verwehren Frauen den Zugang zu Projektmanagement-Schlüsselfunktionen?

- Welche Funktionen nehmen Frauen, die in Projekten arbeiten, wahr?

- Welche Motivation haben Männer/Frauen, eine Projektleitungsfunktion zu übernehmen?

Diesen und anderen Fragen geht das Projekt FIPRO nach.

\section{Projektbeteiligte und Projektpartner}

An dem Projekt arbeiten Studentinnen der Betriebswirtschaft und Wirtschaftsinformatik aus verschiedenen Semestern mit. Die Zahl der teilnehmenden Studentinnen wechselt im Zeitablauf des Projektes. Einige scheiden für die Dauer des Praxissemsters aus, andere haben inzwischen ihr Studium mit dem Diplom erfolgreich abgeschlossen. Im Projektablauf muß daher ein wechselnder Personaleinsatz reibungslos organisiert werden. Insgesamt nahmen bisher 20 Studentinnen an dem Projekt teil. Eine Reihe von Kooperationspartnern unterstützt das Projekt. Einer der wichtigsten Projektpartner ist das brandenburgische Ministerium fuir Wissenschaft, Forschung und Kultur, welches durch seine finanzielle Unterstuitzung die Projektdurchfuihrung sicherstellt. Weitere Projektpartner sind:

- Öffentliche Institutionen

- University of Manchester, Institute of Science and Technology

- Copenhagen Business School

- IPMI Institut für Projektmanagement und Wirtschaftsinformatik, Universität Bremen

- Verbände

- GPM Gesellschaft für Projektmanagement INTERNET Deutschland e.V.

- IPMA International Project Management Association

- APM Association of Project Managers (UK)

- NFP Norwegian Association of Project Management

- SOVNET Russian Project Management Association

- PMA Projektmagement Austria Institut

- AFITEP Association Française du Management de Project

- Unternehmen

- Unternehmensberatungen

- Unternehmen der Automobilbranche

- Unternehmen aus Anlagenbau, Luft- und Raumfahrt

- Unternehmen der Informationstechnologie

- Bauunternehmen

- Banken 
Die genannten Kooperationspartner unterstiitzen das Projekt in der verschiedensten Art und Weise, indem sie Interviewpartner vermitteln, Tagungsgebühren für Studentinnen finanzieren, Ausstellungsfläche auf Kongressen kostenlos zur Verfügung stellen, Adressenmaterial für Befragungen liefern, Diplomarbeiten betreuen und vieles mehr.

\section{Aktionen und Aufgaben im Projekt FIPRO}

Selbstverständlich fallen in dem Projekt die originären Projektfunktionen Planung und Überwachung von Kosten und Terminen an. Von Interesse sind die weiteren Hauptaufgaben, welche den eigentlichen Projektinhalt bilden.

\section{Pilotstudie}

Im Rahmen der Pilotstudie wurden mittels eines halbstandardisierten Fragebogens erste empirische Daten gesammelt. Insgesamt wurden 42 Befragungen durchgeführt. Die Fragebogen wurden an Mitglieder von Dienstleistungsunternehmen, Industrieunternehmen und Bauunternehmen verteilt. Potentielle Befragungsteilnehmer in den beteiligten Unternehmen waren Projektleiter, Projektleiterinnen, Projektmitarbeiter und -mitarbeiterinnen, Personalleiter, Mitglieder des Managements, die über die Vergabe von Projektleitungsfunktionen entscheiden, sowie Projektmanagement-Ausbilder. Die Befragungsteilnehmer wurden gezielt angesprochen, entweder über bestehende eigene Kontakte oder über Kontakte der GPM Gesellschaft für Projektmanagement.

Verschiedene Hypothesen galt es zu überprüfen, z. B.:

- Firmen entsenden ihre Mitarbeiter zu Projektmanagement-Seminaren aus konkreten Projektanlässen und nicht, weil diese Qualifizierung Bestandteil einer gezielten Personalentwicklungsmaßnahme ist.

- Die Übernahme einer Projektführungsposition wird als Bewährungsprobe für zukünftige Leitungsaufgaben in der Linie gesehen und ist somit eine wichti- ge Etappe innerhalb der Laufbahnplanung für Führungskräfte.

Die Ergebnisse hierzu werden in einer gesonderten Ausarbeitung dargestellt.

\section{Internationale Vergleichsstudie}

In Kooperation mit der Universität Manchester und mit Unterstuitzung der IPMA International Project Management Association wird derzeit an einer internationalen Vergleichsstudie mit dem Titel „Organizational culture - gender and the management of project teams" gearbeitet. Die internationale Arbeitsgruppe, an der auch Studentinnen der beteiligten Organisationen mitwirken, hat sich inzwischen über die zu untersuchenden Hypothesen verständigt. Der gemeinsam erarbeitete Fragebogen ist inzwischen getestet und an PM-interessierte versandt. Die Ergebnisse werden im Juni 1996 anläßlich des World Congress on Project Management in Paris vorgestellt.

\section{Dokumentation}

Der Projektstrukturplan veranschaulicht den Umfang der erforderlichen Dokumentationsarbeit. Neben den Ergebnissen aus den verschiedenen empirischen Arbeiten haben die Projektmitglieder inzwischen eine umfangreiche Adressdatenbank aufgebaut. Hierin befinden sich nationale und internationale Ansprechpartner verschiedener Unternehmen zu dem Thema Projektmanagement, Frauen in der Projektarbeit usw. Im Rahmen des Projektes fällt außerdem eine Menge Schriftverkehr an, z. B. Vereinbarungen zu Interviewterminen, Abstimmung mit den Kooperationspartnern, Anfragen von Interessenten.

\section{Praxiskontakte}

Das Projekt wurde und wird auch zukünftig noch auf einer Reihe von nationalen und internationalen Tagungen, Workshops und Kongressen zu dem Thema Projektmangement präsentiert. Gleichzeitig nutzen die Studentinnen die Teilnahme an diesen Tagungen als Gelegen-

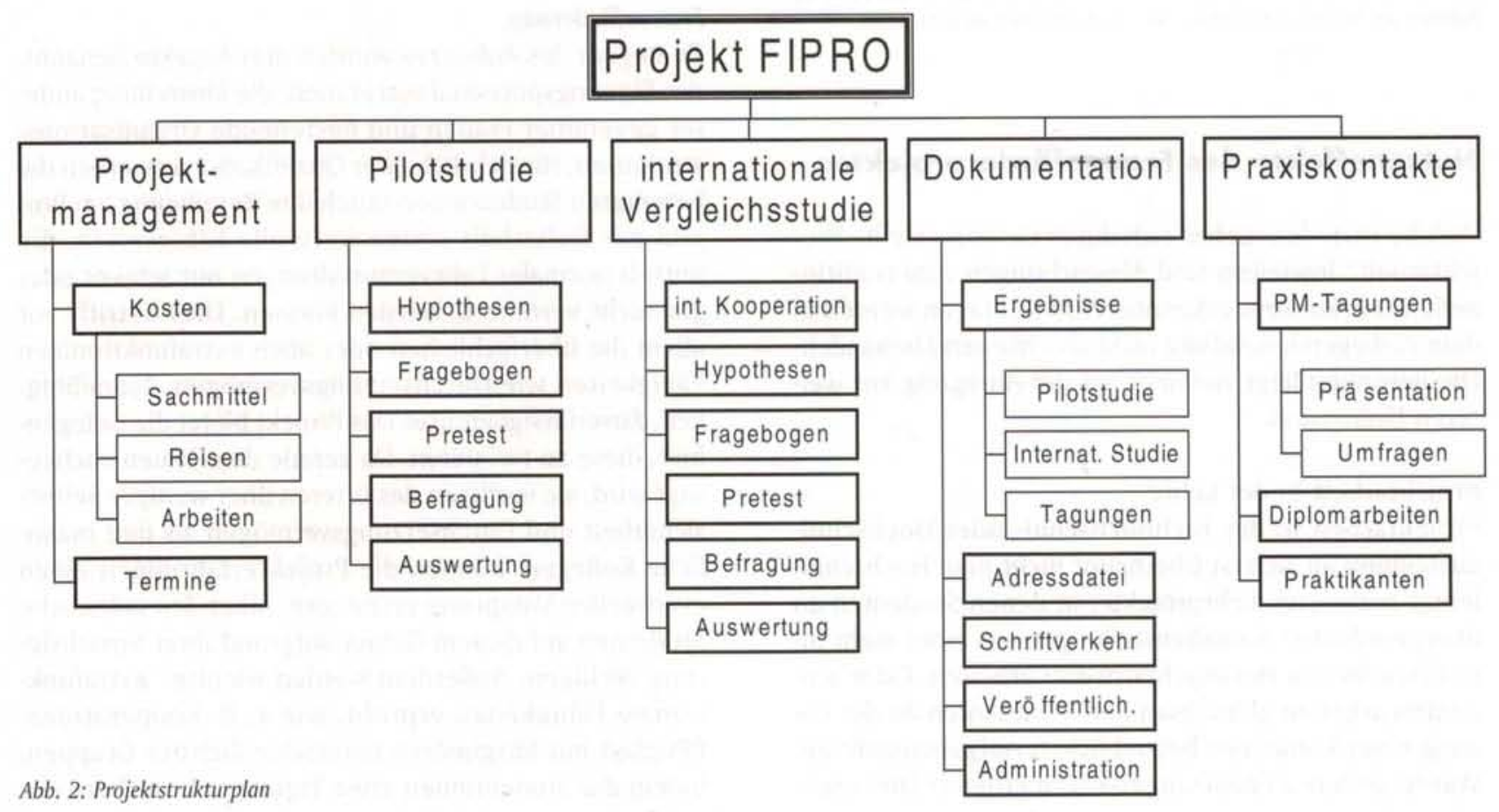


heit, sich zu dem Thema Projektmanagement weiter zu qualifizieren sowie weiteres empirisches Material zu sammeln, z. B. durch kleinere Umfragen, Pretest der Fragebogen, Befragung von Experten.

Bild 3 zeigt die Aktionsorte im Überblick. Die nachfolgende Tabelle beschreibt die Anlässe näher.

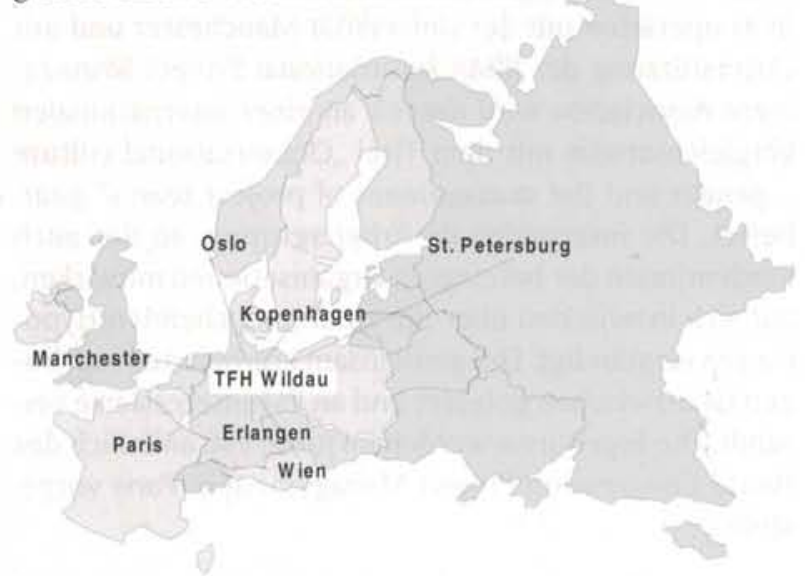

Abb. 3: Nationale und internationale Aktionsorte im Projekt FIPRO

\begin{tabular}{|l|l|l|}
\hline Ort & Zeit & Anlaß \\
\hline Oslo & Juni 1994 & $\begin{array}{l}\text { World Congress on Project Manage- } \\
\text { ment }\end{array}$ \\
\hline Wien & Nov. 1994 & $\begin{array}{l}\text { PM-Tag 94 zum Thema Projekt-Con- } \\
\text { trolling }\end{array}$ \\
\hline Manchester & Nov. 1994 & $\begin{array}{l}\text { Workshop on Women in Project Mana- } \\
\text { gement der APM Association of Pro- } \\
\text { ject Managers }\end{array}$ \\
\hline Kopenhagen & März 1995 & $\begin{array}{l}\text { ERASMUS Intensive Study Week on } \\
\text { Women in Management } \\
\text { mit Beteiligten aus sechs Ländern }\end{array}$ \\
\hline Erlangen & Mai 1995 & $\begin{array}{l}\text { GPM Forum, Jahrestagung der Gesell- } \\
\text { schaft für Projektmanagement }\end{array}$ \\
\hline St. Petersburg & Sept. 1995 & $\begin{array}{l}\text { International Symposim on Project } \\
\text { Management }\end{array}$ \\
\hline Paris & Juni 1996 & World Congress on Project Manage- \\
\hline
\end{tabular}

Abb. 4: Übersicht über Tagungen mentVeranstaltungen, die Studentinnen im Rahmen des Projektes besuchten oder noch besuchen werden

\section{Nułzeneffekłe des Frauenförderprojekłes}

Welche Vorteile ergeben sich durch die vorgestellte Projektarbeit? Inwiefern sind Abweichungen vom traditionellen Lehrkonzept erkennbar? Diese Fragen werden in dem vorliegenden Aufsatz nicht abschließend behandelt. Die Betonung liegt vielmehr auf der Anregung zur weiteren Diskussion.

\section{Projektarbeit in der Lehre}

Projektarbeit in der Fachhochschul- oder Hochschulausbildung an sich ist überhaupt nicht neu. Hochschullehrer realisieren Lehrprojekte, in denen Studenten an übergeordneten Aufgabenstellungen aus einer mehr interdisziplinären Herangehensweise arbeiten. Oder Studenten arbeiten gemeinsam mit Professoren an der Lösung einer konkreten betrieblichen Aufgabenstellung. Was ist anders an dem vorgestellten Projekt? Drei wich- tige Aspekte müssen näher beschrieben werden:

- Praxisnähe,

- Frauenförderung und

- die Rollen von Hochschullehrer und Studenten.

\section{Praxisnähe}

Zunächst lernen die Beteiligten anhand eines praktischen Beispiels den Einsatz von Projektmethoden kennen, insbesondere die Problematik der praktischen Projektsteuerung. Durch den Besuch von Kongressen kommen die Studentinnen sehr intensiv in Kontakt mit Experten aus der Praxis. Sie erleben die Sichtweise der Praktiker zu verschiedenen Fragestellungen in direktem Dialog und auch wie unterschiedlich die Meinungen zu bestimmten Themenbereichen sind, geprägt durch die Branche oder die jeweilige Unternehmenskultur. Kommunikationsprobleme aufgrund verschiedener Begriffsinterpretationen müssen an Ort und Stelle gelöst werden. Die Studentinnen müssen außerdem die Hochschule und das Projekt vor Praktikern präsentieren. Sie übernehmen Verantwortung hinsichtlich der Außenwirkung ihrer Organisation. Der formelle und oftmals internationale Rahmen läßt eine gleichgültige Haltung, wie sie manchmal bei Studenten und Studentinnen in Veranstaltungen durchaus anzutreffen ist, gar nicht erst aufkommen.

Praxisnähe bedeutet auch, Termine per Brief, Fax oder Telefon zu vereinbaren, z. B. für ein Interview. Sparsame Bewirtschaftung der zur Verfügung stehenden Mittel verlangt, daß mehrere Termine koordiniert werden. Zielstrebiges Agieren der Studenten ist gefragt, schließlich sind die kooperierenden Unternehmen primär in die Erledigung ihrer Alltagsaufgaben eingebunden und können einen Telefontermin auch vergessen. Praxisnähe heißt auch, innerhalb der Hochschule den richtigen Umgangston mit den Verantwortlichen für Reisekostenabrechnungen zu treffen. Die Aufzählungen dieser im Projektalltag zu erledigenden Aufgaben ließe sich noch weiter fortführen.

\section{Frauenförderung}

Zu Beginn des Aufsatzes wurden drei Aspekte benannt: das Eignungspotential von Frauen, die Einstellung anderer gegenüber Frauen und bestehende Organisationsstrukturen. Hinsichtlich ihrer Qualifikation erwerben die beteiligten Studentinnen durch ihre Beteiligung am Projekt mit Sicherheit einige wertvolle Fähigkeiten, die mittels normaler Lehrveranstaltungen nur schwer oder gar nicht vermittelt werden können. Dies betrifft vor allem die überfachlichen oder auch extrafunktionalen Fähigkeiten wie Durchsetzungsvermögen, Teamfähigkeit, Zuverlässigkeit usw. Das Projekt bietet die Gelegenheit, diese zu trainieren. Da gerade den Frauen nachgesagt wird, sie verfügen des öfteren uiber weniger Selbstsicherheit und Durchsetzungsvermögen als ihre männliche Kollegen, können die Projekterfahrungen einen eventuellen Vorsprung verringern, über den männliche Studenten auf diesem Gebiet aufgrund ihrer Sozialisierung verfügen. Außerdem werden wichtige extrafunktionale Fähigkeiten erprobt, wie z. B. Kooperationsfähigkeit mit Mitgliedern unterschiedlichster Gruppen, indem die Studentinnen etwa Tagungen besuchen, das 
Projekt vorstellen, Firmen für das Projekt interessieren und Termine für Interviews vereinbaren. Die internationale Kooperation verlangt die Anwendung englischer Sprachkenntnisse, der Umgang mit Menschen anderer Kulturkreise wird geschult. Durch den bereits beschriebenen Personalwechsel im Verlaufe des Projekts nehmen die Studentinnen unterschiedliche soziale Rollen wahr. Erfahrene Projektmitglieder höherer Semester weisen neue Projektmitglieder in die Projektarbeit ein.

Die Begegnung mit anderen Frauen, die als Rollenvorbilder agieren, spielt eine besondere Rolle. Die Existenz von Rollenvorbildern, aber auch die freundliche und unterstützende Aufnahme in der Praxis überhaupt übt eine sehr motivierende Funktion auf die Studentinnen aus. Das Projekt gibt involvierten Praktikern Anlaß zur innerbetrieblichen Diskussion der Thematik. Vorurteile gegenüber Frauen werden unter Umständen abgebaut.

\section{Rolle des Hochschullehrers und der Studenten in der Projektarbeit}

Hochschullehrerin und Studentinnen bilden in dem beschriebenen Projekt eine Aktionseinheit. Sie arbeiten gemeinsam im Team an der Erfüllung einer Aufgabenstellung. Es entsteht eine persönlichere Arbeitsatmosphäre als in der Seminargruppe. Dies unterscheidet sich von der üblichen Rollenverteilung in Lernende und Lehrende. Im Vordergrund steht die Aufgabenlösung. Bei Bedarf werden verschiedene Aspekte näher erläutert. Dies erfolgt jedoch stets an der aktuellen Aufgabe. Klassische Lehrveranstaltungen existieren in dem Projekt nicht. Für die gemeinsame Projektarbeit werden regelmäßige Projektbesprechungen durchgefuihrt, die je nach Bedarf im wöchentlichen oder zweiwöchentlichen Rhythmus erfolgen. Die einzelnen Projektmitglieder sind für bestimmte Aufgaben verantwortlich, z. B. für die Ablage oder das Führen der Action-Items. Im Gegensatz zur traditionellen Lehrveranstaltung müssen komplexe Situationen bewältigt werden, die dem betrieblichen Alltag ähnlicher sind als traditionelle Lehrformen. Hervorzuheben ist, daß Studentinnen verschiedener Semester und Studiengänge zusammenarbeiten. Es ist in der Praxis nicht immer einfach zu realisieren, dennoch, Teamarbeit und der Erwerb überfachlicher Qualifikationen stehen in diesem Projekt eindeutig im Vordergrund.

\section{Offene Fragen}

Zum Abschluß sei auf einige Fragen hingewiesen, die die vorgestellte Projektarbeit für die Hochschulpraxis aufwirft. Projektleistungen können auch mit ein wenig Phantasie nur schwer in das bestehende System der Prüfungsleistungen integriert werden. Versuche diesbezüglich führten in dem vorgestellten Projekt zu eher negativen Erfahrungen. Die Beurteilungssitution im Projekt ist der betrieblichen Praxis ähnlicher als das Erbringen von zurechenbaren Einzelleistungen in Form von Referaten und Klausuren, wie es an Hochschulen üblich ist. Es stellt sich die Frage, inwiefern eine Öffnung der Prüfungsbestimmung oder eine Beurteilungsrelevanz der Projektleistungen überhaupt angestrebt werden kann und soll.

Der zweite Fragenkomplex zielt in Richtung Frauenförderung. Bislang waren an der Projektarbeit nur Stu- dentinnen beteiligt. Ist dies auch für die Zukunft sinnvoll? Bezogen auf die Chancengleichheit bedeutet dies positive Diskriminierung von Frauen. Bis zu welchem Punkt ist dies vor dem Hintergrund der Gleichberechtigung tragbar? Inwieweit wäre die Integration einer Projektarbeit, wie sie hier vorgestellt wurde, in das Studium an Fachhochschulen generell sinnvoll? Wenn ja, unter welchen Bedingungen wäre es machbar und welche Kosten würde dies verursachen?

\section{Anmerkungen}

11] Brodde, Dagmar: Staatlich verordnete Frauenförderung - trojanisches Pferd in deutschen Betrieben?, in: Organisationsentwicklung 1/1990, S. 44-50 (hier: S, 45)

[2] Bundesministerium für Bildung und Wissenschaft: Grund- und Strukturdaten 1993/94, S. 144-146

[3| Domsch, M/ Regnet, E.: Weibliche Fach- und Führungskräfte Wege zur Chancengleichheit, Schriften für Führungskräfte Band 19. Stuttgart 1990

[4] Institute of Management and Remuneration Economics 1994 (Datenbasis: 20.890 Beschäftigte in 330 Organisationen) zitiert nach Davidson , M.J.: Women in Management - Why the Glass Ceiling's not cracking, Unterlage zum Workshop "How effectivs is your organization at teamworking?, 11.11.1994 UMIST Manchester

[5] Rittman, Rosemarie: Mit kühlem Kopf und Powerplay, in: Management Wissen, Heft 7, 1991, S. 22; die Angaben beziehen sich ausschließlich auf die alten Bundesländer

[6] Weinert, A,: Geschlechtsspezifische Unterschiede im Führungsund Leistungsverhalten, in Domsch/Regnet 1990, S. 35-66

[7] Ostner, I.: Zum letzten Male: Anmerkungen zum „weiblichen Arbeitsvermögen" in Krell, G./Osterloh, M. Personalpolitik aus der Sicht von Frauen, München Mering 1993

[8] Eberwein, W./Tholen, J.: Managermentatlität, Frankfurt am Main 1990

[9] Liebrecht, C.H.: Die Frau als Chef, Frankfurt 1985, 1988

[10] Bischoff, Sonja: Frauen zwischen Mann und Macht, Reinbek bei Hamburg 1990

[11] RKW/GPM (Hrsg.): Projektmanagement Fachmann, 2. Aufl. 1994

[12] Huber, Ingrid: Projektmanagement für kleine und mittlere Unternehmen im europäischen Binnenmarkt, Dissertation, IPMI, Universität Bremen 1993

[13] Dworatschek, S./Griesche, D./Meyer, H.: Projektmanagement als wirksames Instrument für ein erfolgreiches Verwaltungsmanagement, in: VOP Fachzeitschrift für öffentliche Verwaltung, Nr. 5. Sept./Okt. 1995, S. 277-288

[14] Dworatschek, Sebastian: Die Entwicklung des Projektmanagement, in: Festschrift für Univ.-Prof. Dr. Dr. h.c. Zimmermann, RWTH Aachen 1994

[15] Gareis, Roland: Management by Projects: Eine Führungsstrategie des projektorientierten Unternehmens, in ders.: Projekt \& Personal, Wien 1991

[16] Dworatschek, S./Meyer, H.: Functions and Qualifications of Project Personnel, Advertisements of vacancies of project staff jobs (empirical study). Proceedings of the INTERNET Conference 2/1993, Cairo 1993

\section{Verfasser}

Prof. Dr. rer. pol. Helga Meyer

Technische Fachhochschule Wildau

Fachbereich Betriebswirtschaft/Wirtschaftsinformatik

Tel. (0 33 75) 507-119 und-124 